\title{
Current and Future Searches for Dark Matter
}

\author{
Daniel A. Bauer \\ Fermi National Accelerator Laboratory, Batavia Illinois 60510 USA
}

\begin{abstract}
Recent experimental data confirms that approximately one quarter of the universe consists of cold dark matter. Particle theories provide natural candidates for this dark matter in the form of either Axions or Weakly Interacting Massive Particles (WIMPs). A growing body of experiments is aimed at direct or indirect detection of particle dark matter. I summarize the current status of these experiments and offer projections of their future sensitivity.
\end{abstract}

Keywords: Dark Matter, WIMP, Axion, Direct Detection, Indirect Detection, CDMS

PACS: $95.35 .+\mathrm{d}, 95.30 . \mathrm{Cq}$

\section{INTRODUCTION}

Within the last few years, the existence of large amounts of non-luminous 'dark' matter within the universe has become well established. It is now known that most of this dark matter is non-baryonic, consisting of particles not predicted by the Standard Model of particle physics. A new generation of experiments is actively searching for these particles using several different techniques. I review the status of current experiments and discuss what the future may bring in the hunt for dark matter.

\section{EVIDENCE FOR THE EXISTENCE OF DARK MATTER}

Independent measurements at many different length scales demonstrate the existence of dark matter [1]. These include rotation curves of galaxies and galaxy clusters, the observed large scale structure of visible matter in the universe, lensing of background galaxies by massive foreground clusters, observations of supernovae, measurements of light element abundance compared with predictions of Big Bang nucleosynthesis and, most recently, measurements of the cosmic microwave background radiation. Taken together these data suggest a universe consisting of roughly $73 \%$ dark energy, $23 \%$ cold, non-baryonic dark matter, $3 \%$ baryonic dark matter, and only 1\% normal luminous matter, as indicated in Figure 1. Clearly one of the keys to understanding the universe is to clarify the nature of both dark energy and dark matter! 


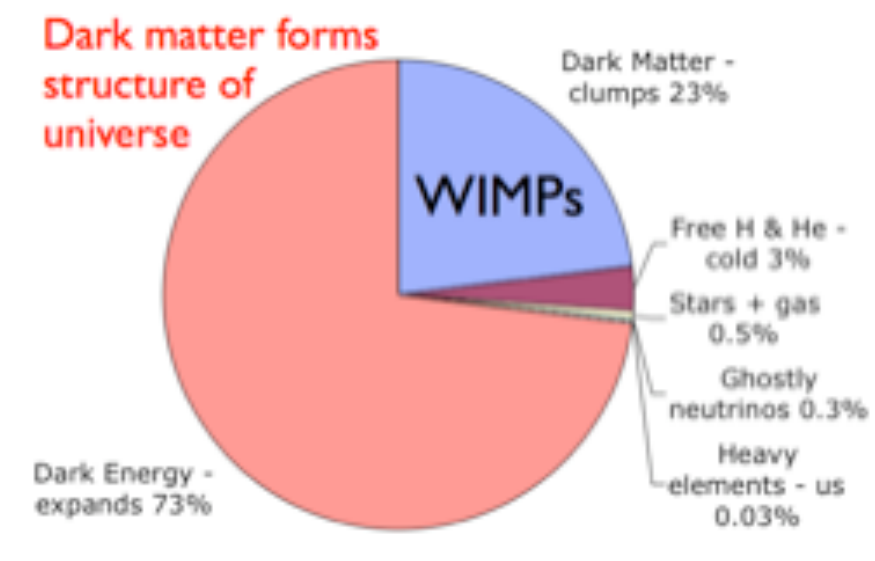

FIGURE 1. Current consensus on the matter and energy content of the universe.

\section{DARK MATTER CANDIDATES}

There is a long list of candidates to explain dark matter [2]. Roughly speaking, they can be divided into three classes: baryonic dark matter, hot dark matter, and cold dark matter.

Baryonic dark matter is normal matter that happens not to produce or absorb a detectable amount of electromagnetic radiation. Sources include cold gas or dust, dead stars, or black holes. Microlensing studies indicate that no more than $20 \%$ of our galaxy's halo could be made of such dark matter. More restrictive limits come from Big Bang Nucleosynthesis, coupled with observations of light element abundances. These constrain baryonic dark matter to be no more than $5 \%$ of the critical density of the universe. This is not enough normal matter to have formed the structures we see, implying that other types of matter must be more important in galaxy formation.

Hot dark matter would consist of particles that were still moving at relativistic speeds at the time radiation and matter decoupled in the early universe. The prime candidate for such matter is neutrinos, which have recently been proven to have mass (albeit not much). It has been demonstrated that hot dark matter cannot produce the observed structure of clusters and galaxies. Furthermore, recent data from neutrinooscillation experiments, tritium-decay measurements, and cosmic microwave background studies constrain neutrino dark matter to be less than $1.5 \%$ of the critical density of the universe.

Cold dark matter consists of particles beyond the Standard Model that were formed in the Big Bang. By the time the expansion of the universe spread them far enough apart that annihilations with other such particles ceased, they were moving at nonrelativistic speeds. It has been known for some time that cold dark matter would clump together gravitationally in the early universe and form the seeds for galaxy formation. There have been a huge number of theories for what these particles are, but two explanations stand out from the crowd due to the fact that they explain more than dark matter. Supersymmetry is a theory that arises naturally in particle physics as an 
explanation of how gravity and the other forces can be unified. One of the particles predicted by supersymmetry is called the lightest stable superpartner. In most versions of the theory, this is a neutral particle (the neutralino) that would have masses in the $10-1000 \mathrm{GeV}$ range and cross sections for interaction with normal matter comparable to those of the weak interaction. Another label for such particles is WIMP, for Weakly Interacting Massive Particle. These particles would be ideal candidates for cold dark matter, since they would have produced in the Big Bang and could be present at a relic abundance that would readily explain the observed amount of dark matter. A second possibility is particles called axions, invented to solve a problem with large QCD CPviolating terms that would lead to unobserved neutron electric dipole moments. As opposed to WIMPS, axions would be very light but also very numerous, and could thus account for the observed dark matter. I will concentrate on these two possibilities for the rest of this paper.

\section{CURRENT SEARCHES FOR DARK MATTER}

I now turn to detection of particle dark matter. Figure 2 illustrates the basic interactions that have been employed by experiments thus far. The only feasible approach for detecting axions has been to search for Primakov conversion, leading to a single photon. WIMPS might be detected indirectly, by observing the products of their annihilation into normal particles. Alternatively, WIMP elastic scattering from nuclei should lead to observable nuclear recoil signatures.
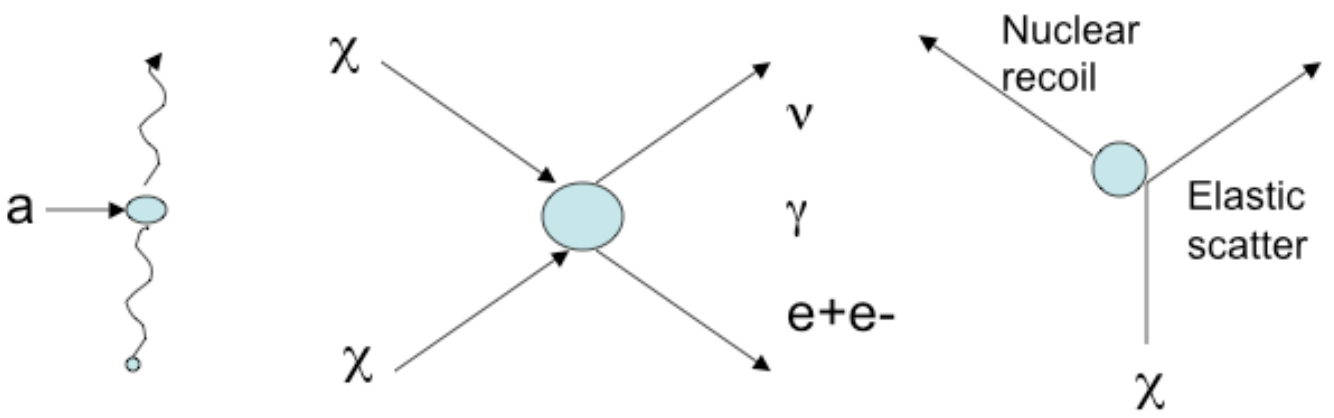

FIGURE 2. Examples of particle dark matter interactions that can be detected. The leftmost diagram shows an axion interacting with a virtual photon from a magnetic field to produce a real photon. The middle diagram illustrates WIMP annihilation to produce pairs of Standard Model particles which can be observed. The rightmost diagram demonstrates a WIMP undergoing an elastic scatter with a nucleus, whose recoil energy can be detected.

\section{Axion Searches}

To be a significant fraction of the cold dark matter in the universe, axions would likely have masses in the range $10^{-6}$ to $10^{-3} \mathrm{eV}$, with coupling strengths to photons in the range $10^{-16}$ to $10^{-14} \mathrm{GeV}^{-1}$. Such small masses and couplings make searches quite challenging.

The main components of all present searches for axions are a large, stable magnetic field to provide a source of virtual photons from which axions can scatter, and a 
sensitive detector with narrow bandwidth to see the resulting real photons. Two large experiments, ADMX and CARRACK (Kyoto), employ tuneable microwave cavities to detect these photons. Another experiment, CAST, looks for x-ray photons and is directional, looking specifically for axions which might have accumulated in the sun.

A recent review [3] summarizes well the current state of axion searches. Although the prospects for covering much of the expected range for dark matter axions look good with the next generation of experiments, the mass range between $10^{-4}$ and $10^{-3}$ $\mathrm{eV}$ may remain elusive for some time.

\section{Indirect Searches for WIMPs}

Prospects for indirect detection [4] of WIMP dark matter rely on two factors. First, the dark matter must be gravitationally concentrated, in places like galactic centers, or perhaps the center of the sun. The larger the density, the greater the rate of annihilations and thus the better the chances of seeing them. Second, the annihilation by-products must reach the Earth without significant deflection and with a rate that distinguishes them from backgrounds. There are many astrophysical sources of neutrinos, gamma rays, and electron-positron pairs that have nothing to do with dark matter and these constitute the 'noise' against which the annihilation signal must be distinguished.

There are three main types of indirect searches. Satellites used for detecting either $511 \mathrm{keV}$ x-rays (from e+e- pair annihilation) or gamma rays from astrophysical sources can be pointed towards the galactic center, searching for an excess over the 'expected' flux. Air Cherenkov or atmospheric fluorescence detectors on the ground can search for showers initiated by high-energy gamma rays with reasonable directionality. Finally, large neutrino detectors can also observe the galactic center.

Two recent satellite observations might be interpreted as signals for WIMP dark matter. The SPI instrument on the Integral Satellite detected a narrow $511 \mathrm{keV}$ line originating from an area roughly the size of the galatic bulge. If this were due to dark matter, however, the WIMP masses would be very light (1-20 MeV), so it is certainly not neutralinos. Meanwhile, the EGRET instrument on the Compton Gamma Ray Observatory has detected an excess of gamma rays from the galactic center. Interpreted as a dark matter annihilation signal, this would imply WIMP-nucleon cross sections around $10^{-43} \mathrm{~cm}^{2}$ and a WIMP mass in the $50-100 \mathrm{GeV}$ range, although these estimates are quite sensitive to the actual structure of the galactic halo near the center. Unfortunately, there are many possible explanations for these excesses other than dark matter. The GLAST satellite, to be launched in 2012, should provide a big improvement in satellite gamma ray sensitivity.

Several large Cherenkov gamma ray detectors are now operational and others are planned. HESS has recently detected $\mathrm{TeV}$ gamma rays from the direction of the galactic center, which might be interpreted as WIMP dark matter with TeV masses, although there are likely other sources for such gamma rays. It will be interesting to see if these results are confirmed and extended by other instruments.

The first generation of neutrino telescopes such as AMANDA has been able to set some limits on dark matter annihilation to neutrinos. As $\mathrm{km}$-scale detectors such as Antares and IceCube come online, these searches become more competitive with 
others, although there will still be the lingering question of whether any signals are due to dark matter or to astrophysical accelerators.

\section{Direct Detection of WIMPs}

All direct detection experiments [5] rely on measurement of the recoil energy deposited by a nucleus recoiling from a WIMP elastic scatter. Since dark matter in the halo of our galaxy moves non-relativistically, these recoil energies are in the 10's of $\mathrm{keV}$ range. If one makes the usual assumption that the dark matter halo is isotropic and isothermal, having a Maxwell-Boltzmann velocity distribution with $\mathrm{V}_{0}=230 \mathrm{~km} / \mathrm{s}$ and density $\rho=0.3 \mathrm{~g} / \mathrm{cm} 3$, the recoil energy distribution is a falling exponential with mean energy of $50 \mathrm{keV}$. Kinematically, the WIMPs most easily detected will be those with mass comparable to that of the target nucleus. If the WIMP-nucleon cross section is of weak interaction strength or less, then the expected detection rate is $<1$ event $/ \mathrm{kg} /$ day.

Obviously to detect such low energy nuclear recoils at such a low rate, heroic measures are needed to suppress backgrounds. Figure 3 shows the important background sources for direct detection experiments. Radioactive decay products can be shielded unless they lie on or near the detectors themselves. Typically, $\mathrm{Pb}$ shielding is used to stop gammas, alphas, and electrons, while polyethylene is used to moderate neutrons. Cosmic ray interactions can be reduced by overburden, which is why all of these experiments are performed in deep underground laboratories, and by active vetoes. The most difficult background is due to neutrons, which produce the same nuclear recoil signature in detectors as do WIMPs. Neutrons produced by cosmic rays in the cavern walls containing the experiment are especially hard to deal with.

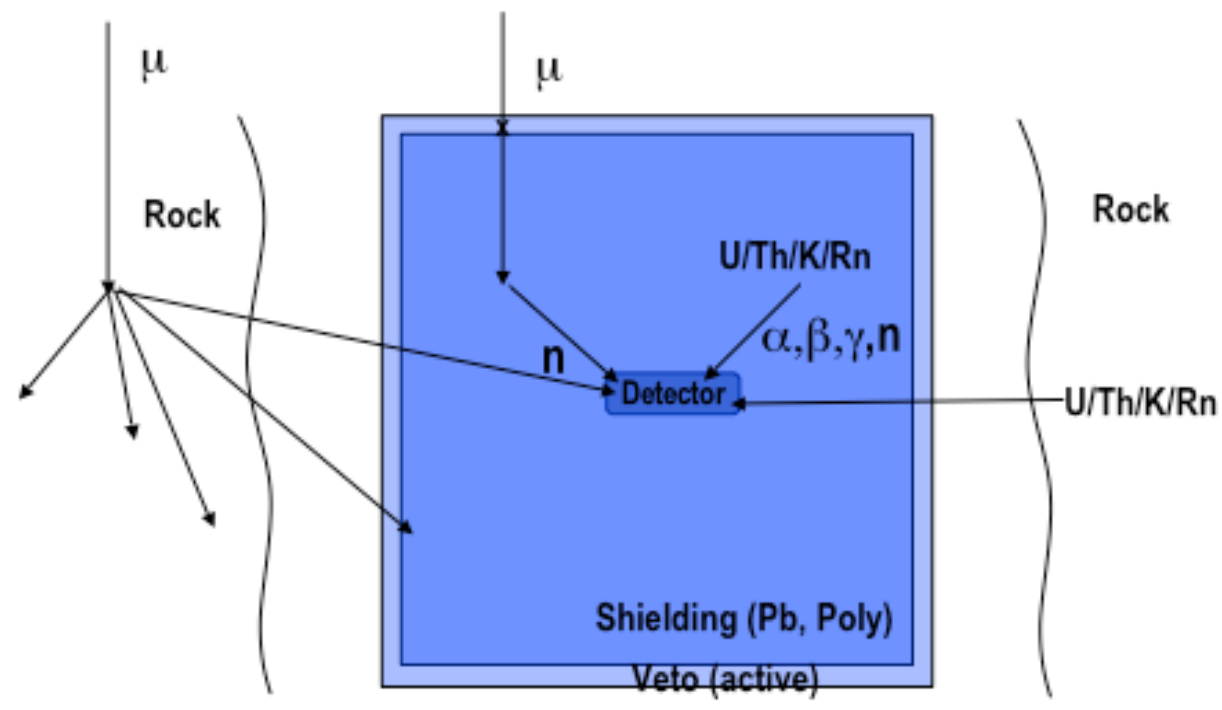

FIGURE 3. Schematic showing sources of backgrounds for direct detection experiments 
Although shielding is necessary, it is not sufficient for the most sensitive experiments. These must also employ active background rejection techniques in the detection process itself, usually by measuring the deposited energy with two different methods that depend on the nature of the recoil (i.e. whether it is electromagnetic or nuclear). An example of such active rejection is shown in Figure 4, from the CDMS experiment. Typical rejection factors against electromagnetic backgrounds now routinely exceed $99 \%$.

Event by event rejection of neutrons is not feasible, but there are statistical means for subtracting remaining neutron backgrounds. Neutrons will frequently scatter more than once in the detectors, whereas WIMPS will not, being weakly-interacting. Also, the WIMP cross section depends either on the nuclear mass or the residual spin of the nucleus, and is thus quite different for typical detector materials like $\mathrm{Si}$ and Ge. However, the neutron cross section does not vary a great deal between these target materials. So a WIMP signal would be characterized by single-scatter events preferentially in the heavier targets or those with the largest spins.
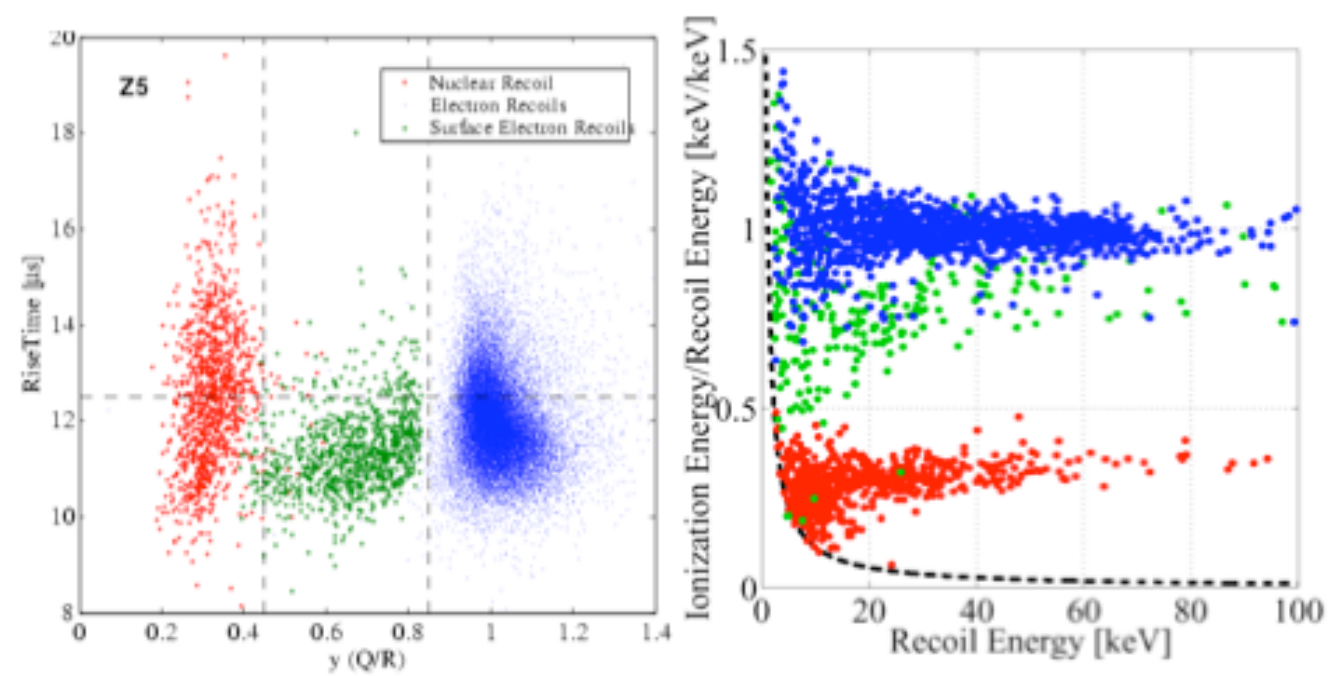

FIGURE 4. Active background discrimination is the key to high sensitivity direct detection searches. This example comes from CDMS. The right-hand plot shows the ratio of ionization to recoil energy (derived from measurement of phonons) plotted versus recoil energy. The horizontal bands near 1 are gammas and betas from a gamma calibration source, while the band near 0.3 shows neutrons from a 252Cf calibration source. There is a clean separation between the electron recoils caused by gammas and betas and nuclear recoils caused by neutrons. Additional separation is shown in the left-hand plot, where the phonon signal risetime is plotted versus ionization yield. From left to right, the vertical bands are those from neutrons, betas, and gammas. Selection of events in the upper left of the plot gives excellent background rejection, with some loss of nuclear recoil acceptance.

Due to the slow velocities of WIMPs, they will interact coherently with nuclei unless such a scaler interaction is dynamically suppressed, in which case their only interaction might be axial, with the spin of the nucleus. Thus the predicted spinindependent cross sections are larger than spin-dependent interactions by the square of the atomic weight of the target material. 


\section{The CDMS Experiment}

The experiment currently leading the field of direct detection is the Cryogenic Dark Matter Search (CDMS) [6], operating in the Soudan Underground Mine in northern Minnesota. CDMS uses ultrapure $\mathrm{Ge}(250 \mathrm{~kg})$ and $\mathrm{Si}(100 \mathrm{~kg})$ crystals, outfitted with sensors to detect both ionization and athermal phonons. As seen in Figure 4, these detectors provide excellent active background rejection, from the ratio of ionization to phonon signals and from the timing of the phonon signal itself. Currently the main background is due to tiny amounts of beta-emitting radioactivity on the surface of the detectors, but demonstrated improvements in phonon timing cuts can keep this background down to $<1$ event for the duration of the experiment. Eventually, a neutron background from residual cosmic ray interactions at Soudan will limit the sensitivity of CDMS at the level of 5-20 events/year.

With current exposures, CDMS has already set upper limits on WIMP-nucleon cross sections of $1.7 \times 10^{-43} \mathrm{~cm}^{2}$ for a WIMP mass of $60 \mathrm{GeV}$, an order of magnitude better than any other experiment. Figure 5 below shows the full limit curve and the region of supersymmetry models already ruled out by the results. Additonal detectors have just been installed, and another order of magnitude improvement in sensitivity is expected after 2 more years of running at Soudan.

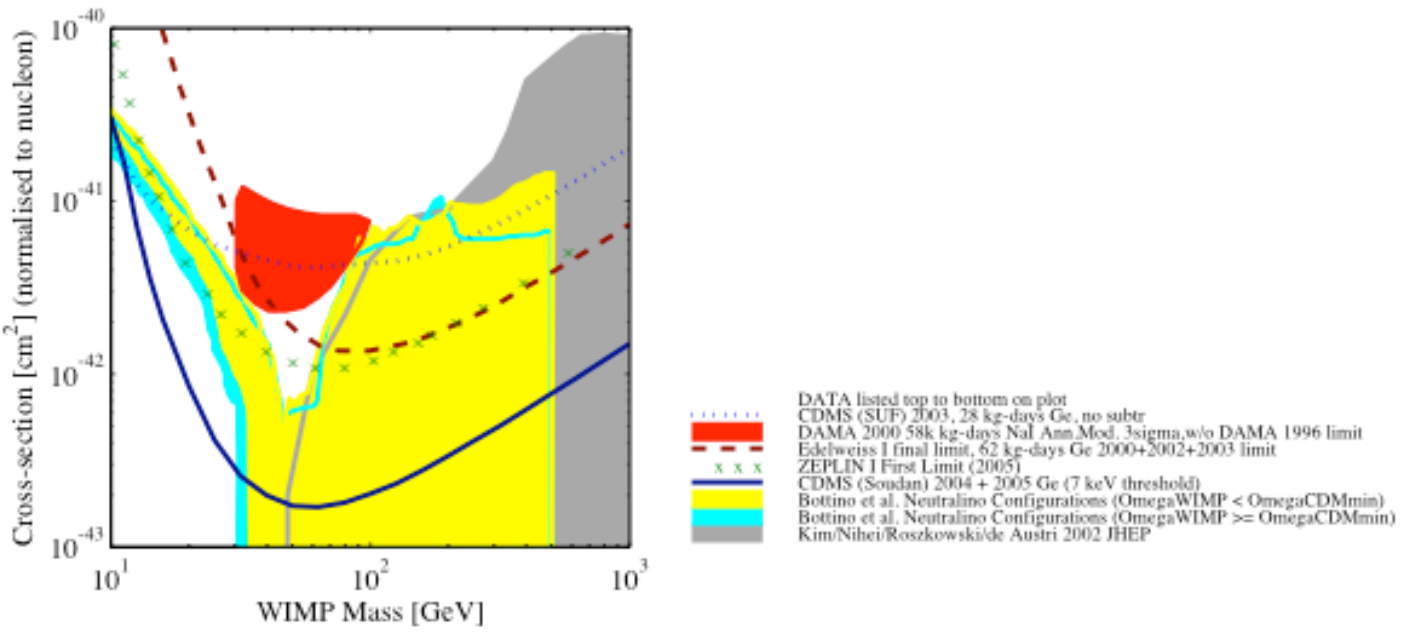

FIGURE 5. Current WIMP dark matter upper limits, plotted as cross section normalized to a single nucleon versus WIMP mass. Note that CDMS limits lead the field by an order of magnitude, and are now ruling out significant parts of the expected parameter space from minimal supersymmetric models

\section{Selected Other Direct Detection Experiments}

A recent review of the field [5] lists the many first- and second-generation direct detection experiments. Given limited space, I have selected only a few with obvious potential to improve the sensitivity of these searches.

EDELWEISS [7] and CRESST [8] are European experiments (based at Modane and Gran Sasso, respectively). Both are cryogenic experiments, with EDELWEISS employing $\mathrm{Ge}$ as the target material to sense both ionization and phonons while CRESST uses CaWO4 equipped to measure scintillation light and phonons. As with CDMS, active rejection is provided by comparing the two different types of signals. 
Both experiments are in a rebuilding phase, deploying detector masses comparable to that of CDMS and intending to start operation in late 2005 or early 2006 . Eventually, the two experiments may merge their technologies into a single experiment called EURECA.

Experiments using liquid noble cryogens as the target material are mostly still in the R\&D phase, although some early results have come from ZEPLIN [9] at Boulby. The XENON experiment has expectations of deploying target masses underground at Gran Sasso of about $10 \mathrm{~kg}$ in 2006 [10]. Liquid Xenon detectors achieve active background discrimination by measuring both ionization and scintillation light, using PMTs immersed in the liquid. A promising approach is to implement a gas phase above the liquid, to boost the ionization signal. If these detectors can achieve energy thresholds of $15-20 \mathrm{keV}$, they will become competitive with semiconductor detectors. Also proposed are large liquid argon [11] and neon [12] experiments.

Another interesting approach in the R\&D stage [13] is the use of heavy liquid (e.g. $\mathrm{CF}_{3} \mathrm{I}$ ) bubble chambers. In principle, these can be made almost completely insensitive to backgrounds from gammas, betas, or minimum-ionizing particles by the correct choice of temperature and pressure, since only heavily-ionizing particles would then be able to initiate bubble formation. Alpha particles and, of course, neutrons would be the remaining backgrounds that must be reduced. Advances in surface preparation have made it possible to maintain such bubble chambers in their superheated state for long periods of time. The challenge will be to extend operational stability to the level of days or weeks. Also, these are intrinsically threshold devices and would give energy information only if run sequentially at different pressures. They are, however, wellsuited for confirmation of a WIMP signal seen by other experiments.

If WIMPS constitute most of the halo of our galaxy, then our solar system is effectively moving through a WIMP 'wind'. For a terrestrial detector, the speed of this wind varies with the time of year because the earth orbits the sun. An experiment with sufficient target mass and extremely good control of systematic errors might be able to measure the small $(\sim 5 \%)$ 'annual modulation' that this would imply for the WIMP detection rate and energy spectrum. Indeed this forms the basis of the only claimed direct detection signal for WIMPS, from the DAMA [14] experiment, a large NaI array deep underground at Gran Sasso. In seven years of data, the experiment sees a $6 \sigma$ annual modulation of rate that seems to correspond to the predicted magnitude and phase for the WIMP wind. However, since essentially all of the effect comes from their lowest energy bin, where many long-term systematic variations are possible, it is reasonable to conclude that the interpretation of this effect as a WIMP signal awaits confirmation from another experiment. An experiment [15] similar in nature to DAMA but using CsI instead of NaI, is underway to confirm or deny the signal. The CDMS experiment, with $\mathrm{x} 10$ better sensitivity, has not seen the WIMP signal that would be implied by DAMA if it were due to spin-independent interactions of WIMPs.

Earth's daily rotation also provides a way to search for the WIMP wind if one can measure the directionality of nuclear recoils. The DRIFT [16] experiment plans to do precisely that, using large, low-pressure time projection chambers loaded with $\mathrm{CS}_{2}$. By drifting negative ions instead of electrons, one can limit diffusion and achieve reasonable precision on the recoiling nucleus direction. Electron recoils can be 
rejected by $\mathrm{dE} / \mathrm{dx}$ and track range measurements. The technology has been demonstrated to work at the Boulby mine. However, it is expensive to achieve sufficient target mass with the low-pressure gas, so a large-scale experiment of this type may await a confirmed WIMP signal from another experiment.

\section{THE FUTURE OF DARK MATTER SEARCHES}

The next generation of direct detection experiments will continue progress towards larger target mass, with better shielding and active discrimination against backgrounds, and greater depth in some cases. Each experiment must face the usual problem that linear improvement in sensitivity with increasing detector mass (M) and running time (T) continues only as long as backgrounds are kept low. Even a wellunderstood background limits one to slower sensitivity gains $(\sqrt{ } \mathrm{MT})$ and an unknown background usually requires changing the experimental technique in some way. All experiments must find ways to deal with the neutron background. Shielding and active neutron vetoes can help, but only depth can reduce the cosmic-ray-induced neutron background sufficiently. The deepest site available is at a depth of 6000 meters (waterequivalent) in the Sudbury mine (Ontario, Canada). This site would provide sufficient reduction of muon flux to make cosmic-ray-induced neutron backgrounds irrelevant even for $1000 \mathrm{~kg}$-scale experiments.

The current generation of direct detection experiments is entering a regime where supersymmetry predicts that a neutralino signal may be seen, and there are intriguing hints from indirect detection experiments as well. Suppose the current level of sensitivity for the CDMS experiment is just on the verge of such a signal. Then, within the next year or two, CDMS should see 5-10 WIMP events, with $<1$ event background. With such a signal, both the WIMP-nucleon cross section and the WIMP mass could be constrained to within an order of magnitude. This would provide valuable information for accelerator experiments at the LHC to look for neutralinos. Conversely, if neutralinos are found first at the LHC, one must still do direct detection experiments (at least one of which would probably be directional) to determine if they constitute the dark matter halo of our galaxy.

Most direct detection experiments are planning to scale to larger detector masses in several stages. Roughly speaking, the next generation of experiments will be in the 10$25 \mathrm{~kg}$ mass range, followed by $100 \mathrm{~kg}$, and eventually $1000 \mathrm{~kg}$ experiments. Figure 6 gives some projections for SuperCDMS [17], planned for the SNOLAB site at Sudbury, but many experiments have similar projections. The shaded regions of the figure show the much larger extent of minimal supersymmetric model predictions that will be accessible to these experiments. The figure also shows the complementarity between direct detection and accelerator experiments for neutralino detection. The largest direct detection experiments will have a somewhat greater reach in both cross section and mass than the LHC experiments. The former will be needed to determine whether the signal really constitutes the dark matter in our galaxy, while the latter will be required to fully explore the neutralino properties. 


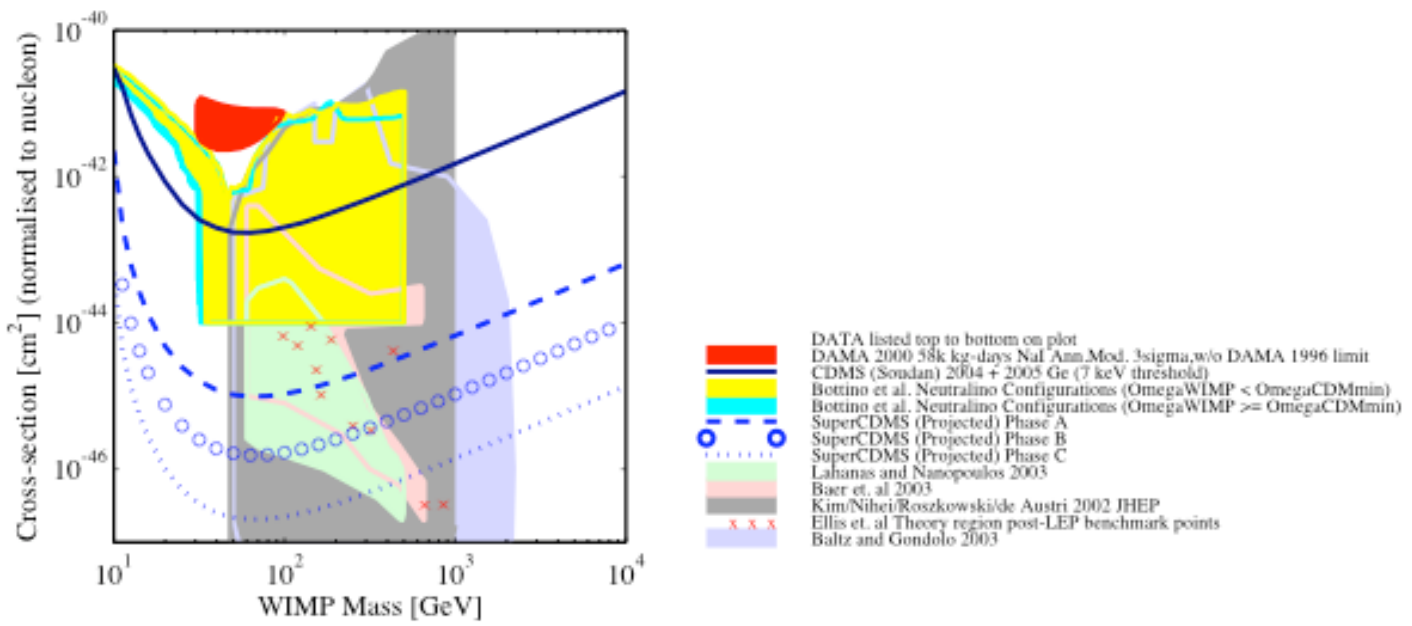

FIGURE 6. Projected reach for the SuperCDMS experiment; many other experiments have similar plans. The shaded regions show a representative sample of predictions from minimal supersymmetry models. The crosses mark studies of the reach of the LHC for neutralino production.

Indirect detection experiments are also getting bigger (in the case of ground-based gamma ray and neutrino telescopes), and more sophisticated (e.g. the GLAST satellite). The challenge for these efforts is to find ways to rule out other interpretations of possible WIMP signals. Axion experiments will continue to refine their detection techniques in both microwave cavity and solar axion searches.

\section{ACKNOWLEDGMENTS}

I would like to acknowledge my CDMS colleagues, as well as those in the rest of the dark matter community, for providing material used herein. I would also like to thank the organizers of PIC2005 for a very interesting and enjoyable conference.

\section{REFERENCES}

1. L. Bergstrom, hep-ph/0312013; hep-ph/0002126 and Rept.Prog.Phys. 63 (2000) 793.

2. L. Roszkowski, hep-ph/0404052.

3. P. Sikivie, hep-ph/0509198 and Pramana 62 (2004) 389-401.

4. G. Bertone, D. Hooper, J. Silk, hep-ph/0404175 and Phys.Rept. 405 (2005) 279-390.

5. R. Gaitskell, Annu. Rev. Nucl. Part. Sci. 2004. 54:315-59.

6. Recent CDMS results can be found in: D.S. Akerib et. al, , astro-ph/0509269; astro-ph/0509259; astroph/0507190; and Phys.Rev.Lett. 93 (2004) 211301.

7. EDELWEISS, astro-ph/0411629.

8. CRESST, astro-ph/0408006,

9. ZEPLIN, astro-ph/0406126.

10. XENON, astro-ph/0502279, astro-ph/0407575.

11. WARP, astro-ph/0405342.

12. CLEAN, astro-ph/0402007.

13. COUPP, astro-ph/0503398.

14. DAMA, astro-ph/0501412 and Int.J.Mod.Phys. D13 (2004) 2127-2160.

15. KIMS, astro-ph/0509080.

16. DRIFT, astro-ph/9905074, astro-ph/9904064, and Phys.Rev. D61 (2000) 101301.

17. SuperCDMS, astro-ph/0502435, astro-ph/0503583.

18.Dark matter limits plots courtesy of R. Gaitskell and V. Mandic. 